\title{
A similarity solution for the flow and heat transfer over a moving permeable flat plate in an external free stream : case of strong injection
}

\begin{abstract}
The previous work of Bachok et al. (Heat Mass Transf. 47:1643-1649, 2011) on the forced convection heat transfer on an isothermal moving surface in an external free stream is extended to the case when fluid injection through the surface, characterized by the parameter $\gamma$, is large. The asymptotic solution derived in this limit shows that the boundary layer has a double region structure, with an inviscid inner region of thickness $O(\gamma)$ and an outer shear layer. Some further aspects of the original problem not treated in Bachok et al. (Heat Mass Transf. 47:1643-1649, 2011) are discussed as well as the analogous problem for a constant surface heat flux, where relatively small injection rates are seen to give rise to large increases in the surface temperature.
\end{abstract}

Keyword: Asymptotic analysis; Forced convection; Heat and mass transfer; Moving surface; Strong injection. 